\title{
A preliminary evaluation of oxidative stress in patients with gastric cancer before chemotherapy
}

Jarosław A. Jakubik ${ }^{1}$, Joanna Kołodziejczyk-Czepas ${ }^{2}$, Magdalena Kędzierska³ ${ }^{3}$ Michał Kaczmarek ${ }^{1}$, Paweł Nowak ${ }^{2}$, Piotr Potemski ${ }^{3}$, Arkadiusz Jeziorski ${ }^{1}$

\author{
${ }^{1}$ Department of Surgical Oncology, Medical University of Lodz, Copernicus Memorial \\ Hospital of Lodz, Lodz, Poland \\ ${ }^{2}$ Department of General Biochemistry, Faculty of Biology and Environmental \\ Protection, University of Lodz, Lodz, Poland \\ ${ }^{3}$ Department of Chemotherapy, Medical University of Lodz, Copernicus Memorial \\ Hospital of Lodz, Lodz, Poland
}

Submitted: 7 September 2018; Accepted: 24 December 2018

Online publication: 18 March 2021

Arch Med Sci 2022; 18 (2): 440-447

DOI: https://doi.org/10.5114/aoms/102344

Copyright $\odot 2022$ Termedia \& Banach

\section{Abstract}

Introduction: Due to an imbalanced redox status, cancer cells generate intrinsically higher levels of reactive oxygen species (ROS) compared to normal cells. Targeting ROS is an important therapeutic strategy for cancer as exemplified by cancer drugs, which induce ROS-dependent synergistic cytotoxicity in gastric cancer cells. The present study was designed to assess the level of selected oxidative stress biomarkers in blood plasma derived from gastric cancer patients.

Material and methods: The study assessed the oxidative/nitrative biomarkers in blood plasma isolated from 51 gastric (adenocarcinoma) cancer patients, compared to a control group of 32 healthy volunteers. Oxidative stress was evaluated using a panel of biomarkers such as plasma protein thiol groups and 3-nitrotyrosine levels as well as indicators of plasma lipid peroxidation, i.e. lipid hydroperoxides $(\mathrm{LOOH})$ and thiobarbituric acid-reactive substances (TBARS). Additionally, the total antioxidant capacity of blood plasma (non-enzymatic capacity of blood plasma, NEAC) was also estimated. Results: Our results showed that patients with gastric cancer had significantly different levels of thiol groups (lower, $p<0.001$ ) and 3-nitrotyrosine (higher, $p<0.0001$ ), LOOH (higher, $p<0.05$ ), TBARS (higher, $p<0.05$ ), NEAC (lower, $p<0.0001$ ), compared to the control group.

Conclusions: The present study indicates considerable oxidative/nitrative stress in gastric cancer patients. Our pilot study shows that not a single marker, but a biomarker panel, may be a more reliable representation of oxidative stress in patients with gastric cancer.

Key words: gastric cancer, blood plasma, oxidative/nitrative stress.

\section{Introduction}

Gastric cancer is an important health problem of contemporary medicine and the third leading cause of cancer-related deaths worldwide. The role of oxidative stress and its contribution to disorders of the gastrointestinal tract have still not been fully elucidated. Extensive intake of non-steroidal anti-inflammatory drugs, stress, alcohol and infections by Helicobacter pylori, which induce a strong pro-inflammatory response of gastric tissue, are listed as the main causes of stomach inflammation, ulcers and oxidative stress

\author{
Corresponding author: \\ Jarosław A. Jakubik PhD \\ Department of Surgical \\ Oncology \\ Medical University \\ of Lodz \\ Lodz, Poland \\ E-mail: ajjak@interia.pl
}


in this organ. However, the etiology and pathophysiology of gastric diseases, including cancer, are more complex and multifactorial. Molecular mechanisms that are responsible for extensive generation of oxidants include activities of numerous pro-oxidant enzymes. The activity of NADPH oxidase, leading to the release of superoxide anion, seems to be of primary importance. For instance, it has been established that this enzyme is involved in resultant gastric mucosal damage [1]. Additionally, an important role in the induction of gastric oxidative stress has also been reported for lipoxygenase (LOX). Studies on pathogenicity of $H$. pylori demonstrated that in human gastric epithelial cells, this bacterium might induce the 5-LOX-catalyzed formation of leukotrienes [2]. Correspondingly, the inflammatory response is stimulated by enzymatic activities of cyclooxygenases (COX-1 and COX-2). Prostaglandins, generated by the arachidonic acid cascade, are mediators of COX-2-derived carcinogenesis. The association of elevated activity of this enzyme with precancerous alterations of the gastrointestinal mucosa was found in Barrett's esophagus and H. pylori-induced gastritis [3]. Furthermore, a close correlation between the expressions of COX-2 and VEGF (a proangiogenic factor) in gastric carcinomas was also observed [4]. Other enzymatic sources of reactive oxygen species (ROS), significantly contributing to oxidative stress in the gastrointestinal tract, are myeloperoxidase, nitric oxide synthase, glucose oxidase and xanthine oxidase [5].

Inflammatory processes involve the recruitment of phagocytes, secreting numerous cytokines and ROS. Besides the antipathogenic action, these inflammatory mediators may also damage surrounding host tissues and lead to oxidative alterations of proteins, lipids and nucleic acids. Oxidative stress enhances the risk of cancer development by a number of mechanisms that are independent of its genotoxicity. Furthermore, oxidative damage to DNA and somatic mutations may result in malignant transformation [6].

The present preliminary study was designed to evaluate the intensity of oxidative stress in the studied group, employing a panel of various biomarkers. The extent of oxidative stress and oxidative damage to blood plasma components was estimated by using a combination of the indicators of protein and lipid oxidation. The efficiency of plasma antioxidant defense in the examined patients was determined as the non-enzymatic antioxidant capacity of blood plasma (NEAC), using the ferric-reducing ability (FRAP) and DPPH-radical-based assays.

\section{Material and methods}

\section{Reagents}

O-phenylenediamine dihydrochloride (SigmaFast OPD peroxidase substrate), 1,1-diphenyl-2-pic- rylhydrazyl (DPPH) and 5,5'-dithio-bis(2-nitrobenzoic) acid (DTNB), 2,4,6-Tris(2-pyridyl)-s-triazine (TPTZ), thiobarbituric acid, and trichloroacetic acid were bought from Sigma-Aldrich (St. Louis, USA).. The OxiSelect Nitrotyrosine ELISA Kit was purchased from Cell Biolabs, Inc. (San Diego, USA). The Pierce BCA Protein Assay Kit was purchased from Thermo Fisher Scientific (Waltham, USA). All other reagents were of analytical grade and were provided by commercial suppliers, e.g. Avantor Performance Materials Poland S.A. (Gliwice, Poland).

\section{The studied group of patients}

Blood samples were taken from 32 healthy volunteers and 51 patients with gastric cancer who were hospitalized in the Department of Oncological Surgery, Medical University of Lodz, Poland. Median age of healthy volunteers was 42 years (range: 25-56 years; mean: 45.7 years; standard deviation: \pm 4.6). There were 51 patients with invasive gastric cancer in the studied group. The final number of patients was 50, because the blood sample from 1 patient underwent hemolysis. Median age of patients was 69 years (range: 43-72 years; mean: 55.1 years; standard deviation: \pm 6.7 ). Clinical profile of the patients (Figure 1) with gastric cancer was estimated according to the $8^{\text {th }}$ edition of AJCC (American Joint Committee on Cancer) TNM: IIIA $-n=6, \| I \mathrm{~B}-n=12$, IIIC $n=18$, IV $n=14)$. Histopathological characteristics: $100 \%$ of gastric cancers were diagnosed as adenocarcinoma. Both groups (patients and healthy volunteers) were maintaining a balanced diet without any antioxidant supplementation. Blood from gastric cancer patients and healthy volunteers was collected onto sodium citrate and immediately centrifuged $(2500 \times \mathrm{g}, 15 \mathrm{~min})$ to obtain plasma. Plasma samples were taken before or 1 to 10 days after surgery. All patients and volunteers expressed their written informed consent for participation in this study. The protocol was accepted by the Committee for Research on Human

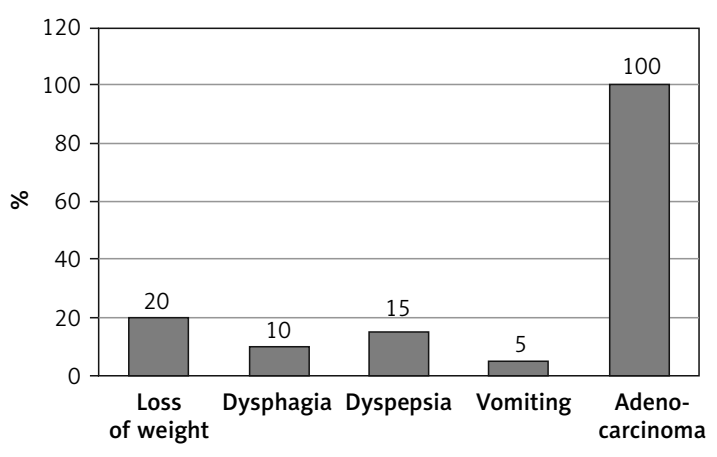

Figure 1. Clinical profile and main symptoms in patients with gastric cancer: loss of weight $-20 \%$ dysphagia $-10 \%$, dyspepsia-15\%, vomiting $-5 \%$. Histopathological characteristics: $100 \%$ of gastric cancer cases were diagnosed as adenocarcinoma 
Subjects of the Medical University of Lodz (KBN N N403 251940).

Determination of the total antioxidant capacity of blood plasma, dependent on the non-enzymatic antioxidants (NEAC)

Determination of the antioxidative capacity using the FRAP assay was carried out according to the method of Benzie and Strain [7]. Before the assay, blood plasma was diluted 10 times with $0.9 \% \mathrm{NaCl}$, and then added to the reagent mixture in a volume ratio of $1: 10: 1: 1$, for plasma, acetate buffer (300 $\mathrm{mM}, \mathrm{pH}$ 3.6), TPTZ $(10 \mathrm{mM}$, in $0.04 \mathrm{M} \mathrm{HCl})$ and $\mathrm{FeCl}_{3}$ $(20 \mathrm{mM}$, in $0.04 \mathrm{M} \mathrm{HCl})$, respectively. The obtained results were expressed as equivalents of $\mathrm{Fe}^{2+}$. The non-enzymatic antioxidant capacity (NEAC) of blood plasma samples was also estimated by 1,1-diphenyl-2-picrylhydrazyl (DPPH) reduction assay.

\section{Detection of thiol groups in plasma proteins}

The amount of protein thiol groups was estimated using 5,5'-dithiobis(2-nitro-benzoic acid) [8]. The concentrations of thiol groups were calculated based on the molar extinction coefficient $\left(13600 \mathrm{M}^{-1} \times \mathrm{cm}^{-1}\right)$.

\section{3-nitrotyrosine immunodetection}

Detection of 3-nitrotyrosine-containing blood plasma was performed by a competitive ELISA meth- od, using the OxiSelect Nitrotyrosine ELISA Kit, according to the protocol provided by the manufacturer.

\section{Determination of lipid peroxidation biomarkers}

Lipid peroxidation was evaluated by measurements of lipid hydroperoxides and thiobarbituric acid-reactive substances (TBARS). Plasma hydroperoxide concentration was determined according to the FOX-1 (ferric-xylenol orange) procedure, described by Gay and Gebicki [9]. TBARS level was also determined spectrophotometrically; absorbance measurements were performed at $532 \mathrm{~nm}$ [10], and the obtained results are expressed as nmoles of TBARS.

\section{Data analysis}

All the values in this study were expressed as mean \pm SD. In order to eliminate uncertain data, Dixon's Q test was performed. The statistical significance of differences between the control group and patients with gastric cancer was done by the Mann-Whitney test using Statistica v. 6.0 (StatSoft Inc.).

\section{Results}

Results obtained in this study indicate significantly enhanced generation of oxidants, leading to oxidative stress in patients with diagnosed gastric adenocarcinoma. Both the protein and lipid components of blood plasma were oxidatively dam-

Table I. Biomarkers of oxidative/nitrative stress in blood plasma isolated from a control group and patients with diagnosed gastric cancer

\begin{tabular}{|c|c|c|c|c|}
\hline \multirow[t]{2}{*}{ Group } & \multicolumn{4}{|c|}{ Oxidative/nitrative stress biomarkers } \\
\hline & $\begin{array}{c}\text { [nmol of thiol } \\
\text { groups/mg of plas- } \\
\text { ma proteins] }\end{array}$ & $\begin{array}{l}\text { [ } \mu \mathrm{mol} \text { of } \mathrm{LOOH} / \mathrm{mg} \\
\text { of plasma proteins] }\end{array}$ & $\begin{array}{c}\text { [nmol of 3-nitrotyro- } \\
\text { sine/mg of plasma } \\
\text { proteins] }\end{array}$ & $\begin{array}{l}\text { [nmol TBARS/mg of } \\
\text { plasma proteins] }\end{array}$ \\
\hline Healthy subjects (A) & $4.99 \pm 0.87$ & $0.09 \pm 0.05$ & $9.21 \pm 8.99$ & $0.016 \pm 0.08$ \\
\hline $\begin{array}{l}\text { Patients with gastric } \\
\text { cancer (B) }\end{array}$ & $\begin{array}{c}2.27 \pm 1.36 \\
(p<0.001-B \text { vs. } A)\end{array}$ & $\begin{array}{c}0.150 \pm 0.11 \\
(p<0.05-B \text { vs. A })\end{array}$ & $\begin{array}{c}22.38 \pm 18.77 \\
(p<0.0001-\mathrm{B} \text { vs. A })\end{array}$ & $\begin{array}{c}0.053 \pm 0.05 \\
(p<0.05-\mathrm{B} \text { vs. A })\end{array}$ \\
\hline
\end{tabular}

Results are expressed as mean \pm SD. Statistical analysis of differences between examined groups was done using the Mann-Whitney test; NS $-p>0.05$

Table II. Summary of differences in levels of oxidative stress biomarkers in blood plasma isolated from 50 gastric (adenocarcinoma) cancer patients, compared to a control group of 32 healthy volunteers

\begin{tabular}{|lc|}
\hline Oxidative/nitrative stress biomarkers & $\begin{array}{c}\text { Healthy subjects (control group) } \\
{[\mathrm{A}]}\end{array}$ \\
\hline 3-nitrotyrosine & $\mathrm{A}<\mathrm{B} ; p<0.001$ \\
\hline Protein thiol groups & $\mathrm{A}<\mathrm{B} ; p<0.001$ \\
\hline Lipid hydroperoxides & $\mathrm{A}>\mathrm{B} ; p<0.05$ \\
\hline Thiobarbituric acid-reactive substances & $\mathrm{A}>\mathrm{B} ; p<0.05$ \\
\hline $\begin{array}{l}\text { Antioxidant capacity of blood plasma measured } \\
\text { by FRAP method }\end{array}$ & $\mathrm{A}>\mathrm{B} ; p>0.05$ \\
\hline $\begin{array}{l}\text { Antioxidant capacity of blood plasma measured } \\
\text { by DPPH-radical based NEAC }\end{array}$ & $\mathrm{A}>\mathrm{B} ; p<0.001$ \\
\hline
\end{tabular}




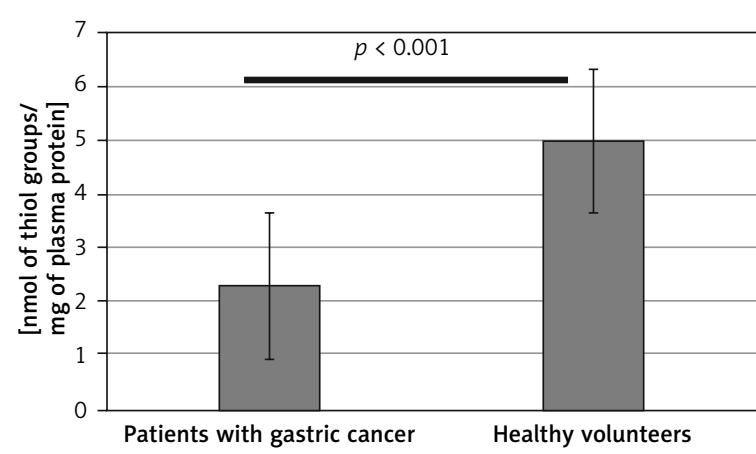

Figure 2. Difference in levels of blood plasma protein thiols in samples from patients before the surgery and chemotherapy and healthy volunteers. Results are expressed as mean $\pm \mathrm{SD}$. Statistical analysis of difference between tested groups was done using the Mann-Whitney test (B vs. A $p<0.001$ )

aged in those subjects (Tables I and II). Our study demonstrated a significant decrease (by about two fold) of blood plasma protein thiol groups $(p<0.001)$ (Figure 2), while the protein 3-nitrotyrosine level was significantly increased ( $p<0.0001)$ (Figure 3). The levels of biomarkers of blood plasma lipid peroxidation such as lipid hydroperoxides $(\mathrm{LOOH})$ and TBARS were also significantly higher than in the control group (Figures 4,5$)$. Additionally, analyses of the antioxidant capacity (dependent on the non-enzymatic antioxidants, NEAC) of blood plasma were performed (Table III). In measurements conducted with both methods (i.e. the FRAP and the DPPH

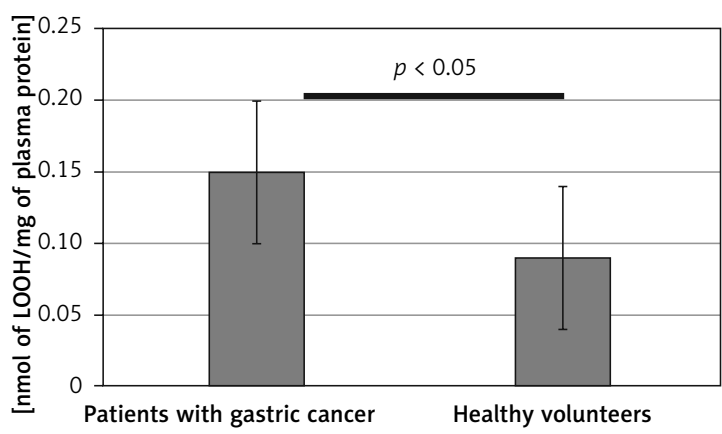

Figure 4. Lipid peroxidation measured by the hydroperoxides $(\mathrm{LOOH})$ level in blood plasma samples from patients before the surgery and chemotherapy and healthy volunteers. Results are expressed as mean \pm SD. Statistical analysis of difference between tested groups was executed using the Mann-Whitney test (B vs. A $p<0.05$ )

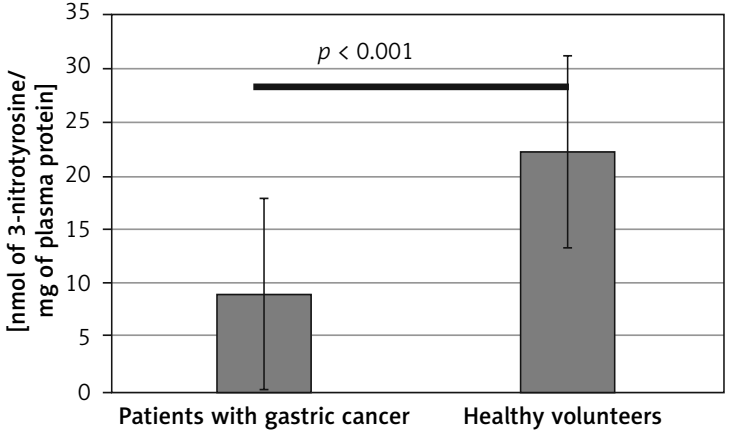

Figure 3. Difference in 3-nitrotyrosine level in blood plasma from patients before the surgery and chemotherapy and healthy volunteers. Results are expressed as mean \pm SD. Statistical analysis of difference between tested groups was done using the Mann-Whitney test (B vs. A $p<0.001$ )

radical-based tests), we observed some decrease of the total antioxidant capacity of blood plasma (Figures 6, 7). However, this reduction of the antioxidant capacity of blood plasma was statistically significant $(p<0.0001)$ only in the case of the $\mathrm{DPPH}$ radical-based test.

\section{Discussion}

The role of oxidative stress in the pathophysiology of gastric cancer, particularly at various stages of tumor growth and metastasis, has been only partly recognized. Only a few investigations have been designed to evaluate the level of oxidative

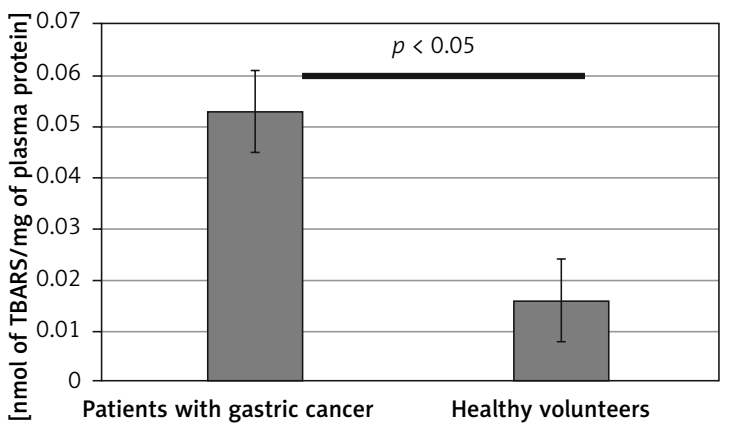

Figure 5. Peroxidation of blood plasma lipids in samples from patients before the surgery and chemotherapy and healthy volunteers, measured as the thiobarbituric acid-reactive substances (TBARS) level. Results are expressed as mean \pm SD. Statistical analysis of difference between tested groups was done using the Mann-Whitney test (B vs. A $p<0.05$ )

Table III. Total antioxidant capacity of blood plasma, dependent on non-enzymatic antioxidants (NEAC) in patients with diagnosed gastric cancer and healthy volunteers (a control group)

\begin{tabular}{|lcc|}
\hline Antioxidant capacity of blood plasma (NEAC) & FRAP $\left[\mathrm{mM} \mathrm{Fe}^{2+}\right]$ & $\begin{array}{c}\text { DPPH-based estimation of NEAC } \\
{[\mu \mathrm{mol} / \mathrm{mg} \text { of plasma protein }]}\end{array}$ \\
\hline Healthy subjects (A) & $0.190 \pm 0.067$ & $0.190 \pm 0.095$ \\
\hline Patients with gastric cancer (B) & $\begin{array}{c}0.179 \pm 0.05 \\
\text { (NS }- \text { B vs. A) }\end{array}$ & $\begin{array}{c}0.140 \pm 0.11 \\
(p<0.001-\text { B vs. A) }\end{array}$ \\
\hline
\end{tabular}

Results are expressed as mean \pm SD. Statistical analysis of differences between examined groups was done using the Mann-Whitney test; NS $-p>0.05$. 


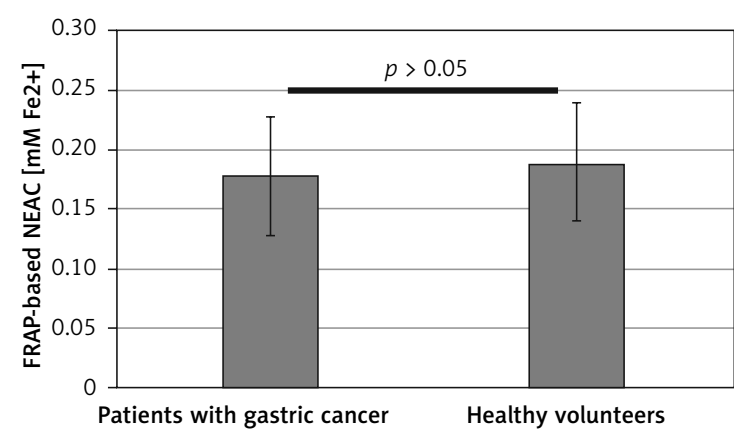

Figure 6. Antioxidant capacity (NEAC) of blood plasma from control group and patients with gastric cancer and healthy volunteers, determined with the FRAP assay. Results are expressed as a mean \pm SD. Statistical analysis of difference between tested groups was done using the Mann-Whitney test (B vs. $A p>0.05$ )

stress biomarkers in patients with gastric cancer. Czygier et al. [11] described results of measurements of the level of granulocyte myeloperoxidase (MPO) and total antioxidant status (TAS) in the plasma of gastric carcinoma patients. In another clinical trial [12], blood plasma activity of glutathione peroxidase was lower in patients with gastric cancer than in the control group; however, measurements of ABTS radical cation reducing ability did not reveal a significant difference between the gastric cancer patients and control subjects. The relation between gastric cancer and oxidative damage to proteins, lipids and DNA was also studied by Ma et al. [13]. Oxidative stress was monitored in blood serum by the determination of different biomarkers of protein oxidation (i.e. carbonyl groups, advanced oxidation protein products (AOPP), and 3-nitrotyrosine (3-NT)) as well as by measurements of DNA damage (8-hydroxydeoxyguanosine level) and lipid peroxidation biomarkers (i.e. malondialdehyde, conjugated dienes, 4-hydroxynonenal, and 8-isoprostaglandin $\mathrm{F}_{2 \alpha}$ ).

The present investigation was designed to evaluate oxidative stress markers in patients with diagnosed gastric adenocarcinoma. Our pilot examination indicates significant oxidative stress in gastric cancer patients before the surgery and chemotherapy. However, not all of the used oxidative stress markers reflected the differences between the studied groups with the same precision. In order to evaluate the intensity of oxidative stress in the examined subjects, we used diverse types of biomarkers that may be easily measured in blood plasma, i.e. 3-nitrotyrosine and plasma thiol groups (protein oxidation biomarkers) as well as lipid hydroperoxides and TBARS (indicators of lipid damage). Additionally, we determined the antioxidant capacity of blood plasma by two different techniques, i.e. FRAP assay and the DPPH-radical based method. While the biomarkers of oxidative damage to proteins and lipids indicated evident-

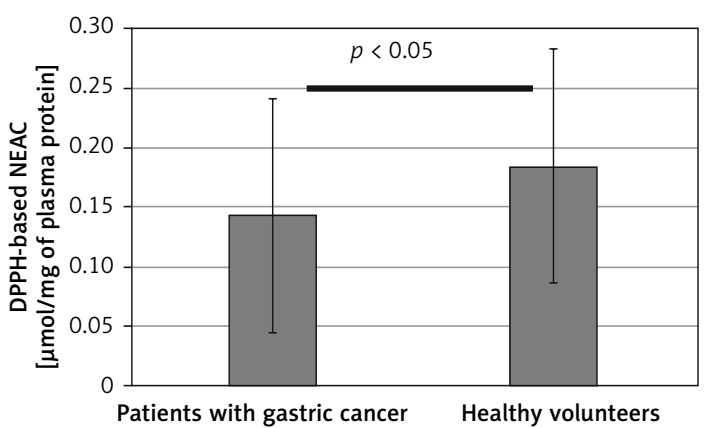

Figure 7. Difference of the antioxidant capacity (NEAC) of blood plasma from patients with gastric cancer and healthy volunteers, measured using the DPPH radical-based assay. Results are expressed as mean \pm SD. Statistical analysis of difference between tested groups was done using the Mann-Whitney test (B vs. A $p<0.05$ )

ly enhanced oxidative stress in the patients, in measurements of the antioxidant capacity evaluated by the FRAP and DPPH-based tests, these differences were weaker. This observation may be a result of different biochemical bases of the used assays. The FRAP assay is based on a chemical reaction with ferrous ions, which may be chelated by blood plasma proteins. Thus, the reaction may be impaired, hindering the detection of differences between the examined samples. For that reason, we suggest that analyses based on the use of this assay should be confirmed by other methods, e.g. DPPH radical-based evaluation of the antioxidant status of blood plasma. A variety of methods is commonly used to evaluate the total antioxidant capacity/total antioxidant status of blood plasma in different cancers. This is one of the basic parameters used to determine oxidative stress. However, results of measurements of the antioxidant capacity of blood plasma are more reliable when they are done using different tests in examinations of the same samples and/or confirmed by other methods. Thus, employing a panel of biomarkers may provide more detailed information on the oxidative stress intensity in patients. These data may be further used for integrated analyses of individual responses to the therapy. However, this issue requires additional studies in the future.

The intensity of oxidative stress may undergo many fluctuations both during the progression of malignancy and in cancer therapy. Low levels of ROS play an important role in proliferation of tumor cells, angiogenesis and metastasis; however, the generation of high levels of various oxidants results in oxidative stress that may induce cell death [14]. Furthermore, in consequence of these changes, the role of antioxidant supplementation during the cancer therapy and in the remission phase has also been reconsidered [15]. Oxidative stress is both a side effect and one of the molecular mechanisms of anticancer treatments. As the 
strongest inducers of oxidative stress, anthracyclines, epipodophyllotoxins, platinum complexes, alkylating agents and camptothecins are mostly listed, whereas nucleotide analogs and taxanes are capable of generating lower levels of oxidants [16]. The action of numerous chemotherapeutic drugs relies on the generation of ROS and induction of apoptosis in cancer cells. Moreover, the recent research on new anticancer strategies includes synergistic action of chemotherapy and oxidative stress. For instance, results of the latest studies on A549 lung cancer cells (Lu et al., 2015) [17] suggested the possibility of using redox responsive nanohybrids as a new strategy for cancer therapy.

The modulation of oxidative stress in cancer cells was considered to be a promising strategy to selectively kill cancer cells, mostly via blocking the activity of superoxide dismutase $[18,19]$. Oxidative stress strongly stimulates the activity of antioxidant enzymes such as superoxide dismutase (SOD) to eliminate superoxide anion and makes the malignant cells more vulnerable to SOD inhibition than normal cells [20]. It has been demonstrated that in human leukemia cells, among others, 2-methoxyestradiol is able to inhibit superoxide dismutase and to induce apoptosis by involving ROS and Akt-dependent signal transduction pathways [21]. On the other hand, it is also noteworthy that the role of oxidative stress in the etiology and pathophysiology of various cancers has been revised in recent years. Although the contributions of inflammation and ROS to carcinogenesis still are undisputable, also other aspects of oxidative stress in cancer diseases should be taken into consideration. ROS are not only cytotoxic molecules, but also modulators of signal transduction. Oxidants are capable of affecting numerous signaling pathways involving mitogen-activated protein kinases (such as ERK1/2, p38 $\alpha$, c-Jun and MEK) and protein kinase $C$ [22]. Furthermore, ROS may also influence transcription factors: NF- $\kappa B$ and AP-1 [23, 24], p53, HIF-1 $\alpha$, PPAR- $\gamma$, $\beta$-catenin/Wnt and Nrf2. As was summarized by Reuter et al., activation of these factors may result in expression of over 500 different genes, including those for growth factors, inflammatory cytokines, chemokines, cell cycle regulatory molecules as well as anti-inflammatory molecules [25, 26]. Additionally, it has been found that oxidative stress may induce the proliferation of cancer cells by inhibiting the tumor suppressor Runt-related transcription factor 3 (RUNX3) [27]. The inactivation of RUNX3, being a result of hypermethylation of its promoter region, is often observed in gastric cancer tissues [28].

Despite the etiology and pathophysiology of various malignancies being complex and multifactorial, epidemiological studies demonstrate an evident association between chronic inflammation and the increased risk of cancer develop- ment. Analyses of protein and lipid biomarkers of oxidative stress demonstrated that both of these components of blood plasma are damaged. From the physiological and pharmacological point of view, oxidative modifications of blood plasma proteins leading to functional consequences may be particularly important. Inflammation, oxidative stress, activation of blood platelets, hypercoagulability and cancer progression are closely linked. Thrombosis was recognized as one of the major complications of malignant diseases as early as in the 1860s. Nowadays, it has been established that the occurrence of many thromboembolic complications is related to clinically occult malignant disease. However, the molecular mechanisms of these disorders still remain inadequately known. Patients with mucin secreting adenocarcinomas of the gastrointestinal tract or ovary are believed to be a particularly high-risk group for the development of hemostatic disorders such as secondary venous thromboembolism [29]. The exposure of the human body to chronic oxidative stress associated with malignant disease may additionally contribute to numerous pathological changes, such as hemostatic disorders. Commonly, clinically evident coagulation disorders are the first sign of malignancy. Disturbances in the hemostatic system induced by tumor growth and metastasis include blood platelet hyperactivity, activation of the coagulation cascade and impaired fibrinolysis. Oxidative damage to blood plasma components (such as protein oxidation and nitration) has been shown to result in various functional consequences [30]. Even very low nitration of fibrinogen ( 45-65 $\mu \mathrm{mol}$ 3-nitrotyrosine/mol tyrosine) may significantly enhance fibrin generation and modify the clot architecture and is capable of increasing the prothrombotic activity of fibrinogen/fibrin [31]. The susceptibility of fibrinolytic proteins to oxidative stress-induced inactivation was also reported [32, 33]. Additionally, blood platelets not only are involved in the metastatic process, but also are able to generate reactive oxygen species during the activation process [34].

In conclusion, the phenomenon of oxidative stress in cancer patients is multifactorial. Our pilot study shows that not a single marker, but a biomarker panel, may be a more reliable representation of oxidative stress in patients with gastric cancer. It is worth continuing further research, which may provide future prognostic markers in the group of cancer patients prior to chemotherapy, surgery or perioperative chemotherapy.

\section{Acknowledgments}

Supported by the grant KBN N N403 251940 from the Medical University of Lodz, Po- 
land. Analyses and/or reagents not included in the above grant were financed from the University of Lodz, Poland (Grant no. 506/1136). J.J, J.K.-C. and M.K. contributed equally to this work.

\section{Conflict of interest}

The authors declare no conflict of interest.

\section{References}

1. Suzuki H, Nishizawa T, Tsugawa H, Mogami S, Hibi T. Roles of oxidative stress in stomach disorders. J Clin Biochem Nutr 2012; 50: 35-9.

2. Go MF, Crowe SE. Virulence and pathogenicity of Helicobacter pylori. Gastroenterol Clin North Am 2000; 29: 649-70.

3. Konturek PC, Kania J, Burnat G, Hahn EG, Konturek SJ. Prostaglandins as mediators of COX-2 derived carcinogenesis in gastrointestinal tract. J Physiol Pharmacol 2005; 56 Suppl: 57-73.

4. Lazăr D, Tăban S, Ardeleanu C, et al. Immunohistochemical expression of the cyclooxygenase-2 (COX-2) in gastric cancer. The correlations with the tumor angiogenesis and patients' survival. Rom J Morphol Embryol 2008; 49: 371-9.

5. Bhattacharyya A, Chattopadhyay R, Mitra S, Crowe SE. Oxidative stress: an essential factor in the pathogenesis of gastrointestinal mucosal diseases. Physiol Rev 2014; 94: 329-54.

6. Dedon PC, Tannenbaum SR. Reactive nitrogen species in the chemical biology of inflammation. Arch Biochem Biophys 2004; 423: 12-22.

7. Benzie IFF, Strain JJ. The ferric reducing ability of plasma (FRAP) as a measure of "antioxidant power": the FRAP assay. Anal Biochem 1996; 239: 70-6.

8. Koster JF, Biemond P, Swaak AJ. Intracellular and extracellular sulphydryl levels in rheumatoid arthritis. Ann Rheum Dis 1986; 45: 44-6.

9. Gay C, Gebicki JM. A critical evaluation of the effect of sorbitol on the ferric-xylenol orange hydroperoxide assay. Anal Biochem 2000; 284: 217-20.

10. Wachowicz B. Adenine nucleotides in thrombocytes of birds. Cell Biochem Funct 1984; 2: 167-70.

11. Czygier M, Kamocki Z, Ławicki S, Szmitkowski M. The plasma level of myeloperoxidase (MPO) and total antioxidant status (TAS) in gastric cancer patients after surgery. Przegl Lek 2010; 67: 443-5.

12. Baroudi O, Ben Younes S, Mezlini A, et al. Status of antioxidant enzyme: glutathione peroxidase and total polyphenol level in plasma of Tunisian patients suffering from colorectal and gastric cancer: interaction with clinical outcome. Med Oncol 2013; 30: 731.

13. Ma Y, Zhang L, Rong S, et al. Relation between gastric cancer and protein oxidation, DNA damage, and lipid peroxidation. Oxid Med Cell Longev 2013; 2013: 543760.

14. Kardeh S, Ashkani-Esfahani S, Alizadeh AM. Paradoxical action of reactive oxygen species in creation and therapy of cancer. Eur J Pharmacol 2014; 735: 150-68.

15. Milkovic L, Siems W, Siems R, Zarkovic N. Oxidative stress and antioxidants in carcinogenesis and integrative therapy of cancer. Curr Pharm Des 2014; 20: 6529-42.

16. Conklin KA. Chemotherapy-associated oxidative stress: Impact on chemotherapeutic effectiveness. Integr Cancer Ther 2004; 3: 294-300.
17. Lu HY, Chang YJ, Fan NC, et al. Synergism through combination of chemotherapy and oxidative stress-induced autophagy in A549 lung cancer cells using redox-responsive nanohybrids: a new strategy for cancer therapy. Biomaterials 2015; 42: 30-41.

18. Huang P, Feng L, Oldham EA, Keating MJ, Plunkett W. Superoxide dismutase as a target for the selective killing of cancer cells. Nature 2000; 407: 390-5.

19. Hileman EA, Achanta G, Huang P. Superoxide dismutase: an emerging target for cancer therapeutics. Expert Opin Ther Targets 2001; 5: 697-710.

20. Hileman EO, Liu J, Albitar M, Keating MJ, Huang P. Intrinsic oxidative stress in cancer cells: a biochemical basis for therapeutic selectivity. Cancer Chemother Pharmacol 2004; 53: 209-19.

21. Gao N, Rahmani M, Dent P, Grant S. 2-methoxyestradiol-induced apoptosis in human leukemia cells proceeds through a reactive oxygen species and Akt-dependent process. Oncogene 2005; 24: 3797-809.

22. Sosa V, Molinéa T, Somoza R, Paciucci R, Kondoh H, Leonart ME. Oxidative stress and cancer: an overview. Ageing Res Rev 2013; 12: 376-90.

23. Wang $\mathrm{Y}$, Huang $\mathrm{X}$, Cang $\mathrm{H}$, et al. The endogenous reactive oxygen species promote NF-kappa B activation by targeting on activation of NF-kappa B-inducing kinase in oral squamous carcinoma cells. Free Radic Res 2007; 41: 963-71.

24. Bubici C, Papa S, Dean K, Franzoso G. Mutual crosstalk between reactive oxygen species and nuclear factor-kappa B: molecular basis and biological significance. Oncogene 2006; 25: 6731-48.

25. Reuter S, Gupta SC, Chaturvedi MM, Aggarwal BB. Oxidative stress, inflammation, and cancer: How are they linked? Free Radic Biol Med 2010; 49: 1603-16.

26. Zeng C, Liu Z, Zhang J, et al. Functions of the AP- $2 \alpha$ gene in activating apoptosis and inhibiting proliferation of gastric cancer cells both in vitro and in vivo. Arch Med Sci 2017; 13: 1255-61.

27. Kang KA, Kim KC, Bae SC, Hyun JW. Oxidative stress induces proliferation of colorectal cancer cells by inhibiting RUNX3 and activating the Akt signaling pathway. Int J Oncol 2013; 43: 1511-6.

28. Li QL, Ito K, Sakakura C, et al. Causal relationship between the loss of RUNX3 expression and gastric cancer. Cell 2002; 109: 113-24.

29. Rickles FR, Abe MSK. The role of the hemostatic system in tumor growth, metastasis, and angiogenesis: tissue factor is a bifunctional molecule capable of inducing both fibrin deposition and angiogenesis in cancer. Int J Hematol 2001; 73: 145-50.

30. Nowak P, Zbikowska HM, Ponczek M, Kolodziejczyk J, Wachowicz B. Different vulnerability of fibrinogen subunits to oxidative/nitrative modifications induced by peroxynitrite: functional consequences. Thromb Res 2007; 121: 163-74.

31. Vadseth C, Souza JM, Thomson L, et al. Pro-thrombotic state induced by post-translational modification of fibrinogen by reactive nitrogen species. J Biol Chem 2004; 279: 8820-6.

32. Hathuc C, Hermo R, Schulze J, Gugliucci A. Nitration of human plasminogen by RAW 264.7 macrophages reduces streptokinase-induced plasmin activity. Clin Chem Lab Med 2006; 2: 213-9. 
33. Nowak P, Kolodziejczyk J, Wachowicz B. Peroxynitrite and fibrinolytic system: the effect of peroxynitrite on plasmin activity. Mol Cell Biochem 2004; 267: 141-6.

34. Redondo PC, Jardin I, Hernández-Cruz JM, Pariente JA, Salido GM, Rosado JA. Hydrogen peroxide and peroxynitrite enhance $\mathrm{Ca} 2+$ mobilization and aggregation in platelets from type 2 diabetic patients. Biochem Biophys Res Commun 2005; 333: 794-802. 